\title{
DESCRIPCIÓN DE DOS NUEVAS ESPECIES CAVERNÍCOLAS DE TRICHONISCINAE VERHOEFF, 1908 DE LA CORDILLERA CANTÁBRICA (CRUSTACEA: ISOPODA: TRICHONISCIDAE)
}

\author{
Julio Cifuentes ${ }^{1} \&$ Carlos E. Prieto ${ }^{2}$ \\ ${ }^{1}$ Departamento de Biología (Zoología), Facultad de Ciencias, Universidad Autónoma de Madrid,28049 Cantoblanco, Madrid, España. \\ Email: jcifcol@gmail.com - ORCID-iD: https://orcid.org/0000-0002-9569-6973 \\ ${ }^{2}$ Departamento de Zoología y Biología Celular Animal, Facultad de Ciencia y Tecnología, Universidad del País Vasco, POBox 644, 48080 Bilbao, España. \\ Email: carlos.prieto@ehu.eus - ORCID-iD: https://orcid.org/0000-0003-2064-4842
}

\begin{abstract}
RESUMEN
Dos nuevas especies cavernícolas de la Península lbérica pertenecientes a la subfamilia Trichoniscinae Verhoeff, 1908, han sido descubiertas gracias al estudio de los isópodos terrestres de la Colección de Fauna Cavernícola de la Universidad del País Vasco. Trichoniscoides cantabricus $\mathrm{n}$. sp. se describe de las cavidades del Miera-Asón, estando presente también en otras tres áreas kársticas de Cantabria y una cavidad del norte de Burgos. Troglonethes fonsocalvoi n. sp. se describe de las cavidades de Montes de Triano (Bizkaia) y está presente también en Sierra Salvada y los macizos de Jorrios-Alén, Gorbea.
\end{abstract}

$$
\text { http://zoobank.org/urn:Isid:zoobank.org:pub:5DE67D90-805E-458D-BBFA-04DF244FBD68 }
$$

Palabras clave: Crustacea, Isopoda, Trichoniscidae, taxonomía, fauna cavernícola, península lbérica.

\begin{abstract}
Description of two new cavernicolous species of Trichoniscinae Verhoeff, 1908 from the Cantabrian mountains (Crustacea: Isopoda: Trichoniscidae)

Two new cavernicolous species of the lberian Peninsula, belonging to the sub-family Trichoniscinae Verhoeff, 1908, have been discovered through the study of the terrestrial isopods present in the "Colección de Fauna Cavernícola de la Universidad del País Vasco". Trichoniscoides cantabricus n. sp. is described from Miera-Asón river system cavities, being present in another 3 karstic environments in Cantabria, and another cavity in the north of Burgos. Troglonethes fonsocalvoi n. sp. is described in the Montes de Triano cavities (Bizkaia) and it is also present at Sierra Salvada and the Jorrios-Alén and Gorbea massifs.
\end{abstract}

Key words: Crustacea, Isopoda, Trichoniscidae, taxonomy, cave fauna, Iberian Peninsula.

Recibido/Received: 6/04/2020; Aceptado/Accepted: 7/09/2020; Publicado en línea/Published online: 05/05/2021

Cómo citar este artículo/Citation: Cifuentes, J. \& Prieto, C. E. 2021. Descripción de dos nuevas especies cavernícolas de Trichoniscinae Verhoeff, 1908 de la Cordillera Cantábrica (Crustacea: Isopoda: Trichoniscidae). Grael/sia, 77(1): e124. https://doi.org/10.3989/graellsia.2021.v77.275

Copyright: (C) 2021 SAM \& CSIC. Este es un artículo de acceso abierto distribuido bajo los términos de una licencia de uso y distribución Creative Commons Reconocimiento 4.0 Internacional (CC BY 4.0).

\section{Introducción}

Los isópodos terrestres (suborden Oniscidea) son uno de los elementos más comunes y conspicuos de la biota subterránea, siendo la familia Trichoniscidae Sars, 1899 la más ampliamente representada en el medio subterráneo (Taiti, 2004). En la región Iberobalear están representados 18 géneros (Schmalfuss, 2003), casi todos ellos con algún representante cavernícola y cinco de ellos endémicos y troglobios. En el área cantábrica, la familia está representada por siete géneros (Vandel, 1960; Bellés, 1987): Cantabroniscus Vandel, 1965, monotípico para C. primitivus Vandel, 1965, distribuido por Cantabria y norte de Burgos y uno de 
los escasos representantes acuáticos de la familia; $E s$ cualdoniscus Vandel, 1948 con la especie troglobia E. coiffaiti Vandel, 1948 en el extremo occidental de Pirineos, y otra endógea E. triocellatus Vandel, 1948 en la misma región; Haplophthalmus Schöbl, 1861 con H. asturicus Vandel, 1952 conocido sólo de una cueva asturiana; Metatrichoniscoides Vandel, 1942 con el endógeo M. fouresi euskariensis Vandel, 1957 en los Pirineos occidentales franceses; Miktoniscus Kesselyák, 1930 con la epigea litoral M. bisetosus Vandel, 1946 en Asturias; Oritoniscus Racovitza, 1908 con dos especies humícolas, $O$. remyi Dalens, 1964 y $O$. simplex Vandel, 1957, y finalmente el género Trichoniscoides Sars, 1899, ampliamente representado.

Las especies del género Trichoniscoides son mayoritariamente cavernícolas, seis de las doce especies presentes en la Cornisa Cantábrica se conocen exclusivamente del medio subterráneo, cinco son especies epigeas acantonadas entre la Sierra de Ancares y Picos de Europa y la última, T. lusitanus Vandel, 1946 se extiende desde el noroeste de Portugal hasta Cantabria (Vandel, 1946, 1952; Cifuentes, 2019). Las áreas de distribución conocidas de las especies de este género, especialmente las cavernícolas, son en general reducidas, hecho ya señalado por Vandel (1946, 1952, 1960), y en ocasiones limitadas a las localidades que sirvieron para describirlas. Para la región cantábrica, T. cavernicola (Budde-Lund, 1885) es la excepción por su extensa área de distribución desde Asturias y norte de León hasta Gipuzkoa y norte de Navarra, y por el sur hasta Soria (Vandel, 1946, 1952; Schmölzer, 1971; Cifuentes, 2019). A pesar de que ese área solapa las de las otras especies cantábricas, hasta el momento no se han citado cavidades en las que T. cavernicola coexista con alguna de ellas.

El estudio de la fauna peninsular ibérica de isópodos triconíscidos representada en la Colección de Fauna Cavernícola de la Universidad del País Vasco, objeto de este trabajo, ha permitido hasta el presente, elevar a 29 el número de especies conocidas del género Trichoniscoides en la península ibérica, lo que supone el $62 \%$ de todas las especies válidas descritas de este género, poniendo de manifiesto la gran riqueza en especies de la fauna de Trichoniscoides ibérica.

Esta diversidad también se refleja en el hallazgo de una nueva especie del género endémico Troglonethes Cruz, 1989, previamente desconocido de la región cantábrica ya que sólo se conocía la alicantina especie típica T. aurouxi Cruz, 1989 y dos especies recientemente descritas de Portugal (Reboleira et al., 2015).

El objetivo de este trabajo es por tanto describir dos nuevas especies para la ciencia de estos géneros, y discutir las diferencias con especies próximas que permitan una fácil separación de ellas. Dado que ya se había facilitado (Cifuentes, 2019) una clave de las especies peninsulares de Trichoniscoides, en este caso se facilita una clave para las especies del género Troglonethes, la cual solamente es válida para los machos.

\section{Material y métodos}

Los ejemplares que se han utilizado para la descripción de las dos especies proceden de la Colección de Fauna Cavernícola del Departamento de Zoología de la Universidad del País Vasco (CFC/ZUPV), aunque los holotipos y algunos paratipos se han depositado en el Museo Nacional de Ciencias Naturales (MNCN).

También se han estudiado los ejemplares tipo de T. aurouxi depositados en la colección de la Universidad de Barcelona: Valencia, Serra, sierra Calderona, avenc Garbí, V-1980, 2 ○ึે, Auroux leg., A. Cruz col., CRBA86606; cova de l'Alt del Pi o de les Aranyes, 20-I-1990, 1 ồ y 1 ㅇ, J. Jornet leg., A. Cruz col., CRBA-86608.

Las preparaciones microscópicas se han realizado con la resina sintética DMHF (dimetil hidantoina formaldehído). Para facilitar la identificación de estas nuevas especies, se han incluido tanto imágenes procedentes de fotografías como dibujos digitalizados a partir de las fotografías con el editor gráfico InKscape (https://inkscape.org/es/).

\section{Resultados}

\section{Trichoniscoides cantabricus $\mathrm{n}$. $\mathrm{sp}$.}

urn:Isid:zoobank.org:act:1F6944A5-14A9-401E-8CC8-99CAA130AD08

Material típico. Holotipo, ô, Cantabria, Arredondo, Bustablado, cueva del Molino (30TVN4843292186, 239 msm), 28-VIII-2019, C. Prieto y A. Sendra leg., MNCN 20. 04/12330 [ex CFC/ZUPV 5628].

Paratipos. Burgos, Guareña, Merindad de Sotoscueva, S. Martín de Sotoscueva, cueva Racino (30TVN4398263355, 827msm), 26-XI-2017, 1 ô, C. Prieto y J. Fernández leg., CFC/ ZUPV 5021. Cantabria, Arredondo, Bustablado, cueva del Molino (30TVN4843292186, $230 \mathrm{msm}$ ), 28-VIII-2019, 1 के y 1 ㅇ, C. Prieto y A. Sendra leg., MNCN 20. 04/12331 [ex CFC/ ZUPV 5628]; 28-VIII-2019, 1 $\hat{\jmath}$, C. Prieto y A. Sendra leg., CFC/ZUPV 5628. Arredondo, Socueva, cueva de San Juan de

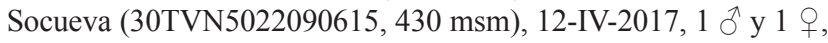
C. Prieto, S. Quiñonero, J. Ruiz-Cobo y A. Alonso leg., CFC/ ZUPV 4718. Castro Urdiales, Sámano, cueva de La Lastrilla (30TVP7922100772, $60 \mathrm{msm}$ ), 18-V-2014, 1 \%, C. Prieto, A. Calvo y E. Ogando "Zape" leg., CFC/ZUPV 2333. Miera, Ajanedo, cueva de La Puntida (30TVN4233889803, $500 \mathrm{msm}$ ), 12-X-2015, 1 §̊, C. Prieto y A. Calvo leg., CFC/ZUPV 3037. Puente Viesgo, cueva Sumidero (Sistema Búho) (30TVN1889994082, 400 msm), 22-IV-2012, 1 †, C. Prieto y J. Fernández leg., CFC/ ZUPV 667. Puente Viesgo, cueva del Búho (30TVN1865493982, 420 msm), 27-III-2018, 1 ㅇ, C. Prieto, S. Quiñonero, J. Ruíz-Cobo y A. Alonso leg., CFC/ZUPV 5173. Puente Viesgo, Penilla, cueva de Pis o Piz (30TVN2403393124, 125 msm), 27-XI-2016, 1 ô, C. Prieto y J. Fernández leg., CFC/ZUPV 4471. Ruesga, Matienzo, cueva de la Cubija o de Marcos (30TVN5028896324, $269 \mathrm{msm}$ ), 19-VII-2016, 1 + , C. Prieto, S. Quiñonero y J. Ruiz-Cobo, CFC/ ZUPV 4059. San Miguel de Aras, Caburrao, cueva de Cobrantes (30TVN5712496422, 145 msm), 17-VIII-1968, 1 ô, GE. Esparta leg., CFC/ZUPV 2153. San Roque de Riomiera, Merilla, cueva de Covallarco (30TVN4041289565, $420 \mathrm{msm}$ ), 18-VI-2016, 1 §, C. Prieto, J. Fernández y Marcos, CFC/ZUPV 3973. San Roque 
de Riomiera, Porracolina, sima PO-153 (30TVN4392886570, $585 \mathrm{msm}$ ), 12-XI-2016, 1 \&, C. Prieto, M. Gutiérrez y J.A. Noriega, CFC/ZUPV 4201. Udías, cueva de Udías (mina Sel del Haya (30TVP0043800328, 222 msm), 16-I-2016, 1 đ̄ y 7 우, C. Prieto, J. Fernández y M. Gutiérrez leg., CFC/ZUPV 3430. Udías, cuevamina La Buenita (30TVP999993, $155 \mathrm{msm}$ ), 2-II-2020, 3 के के y 3 우우, C. Prieto y J. Fernández leg., CFC/ZUPV 5837.

En total se han estudiado 14 machos y 16 hembras.

Etimología. El nombre específico alude a Cantabria, Comunidad del norte de España.

Diagnosis. Tegumento con fuertes granulaciones. Flagelo antenal formado por tres artejos. El exopodito de pleópodo I del macho es de forma cuadrangular, con el borde externo redondeado y el interno recto, $\mathrm{y}$ tiene dos tallos ciliados casi iguales. El endopodito del pleópodo I del macho es triangular, con un lóbulo distal redondeado. El exopodito del pleópodo II del macho es triangular, con una punta interna muy desarrollada, provista de un fuerte tallo.

\section{DESCRIPCIÓN}

Talla máxima observada: $3 \times 0,7 \mathrm{~mm}$ sin los urópodos.

Coloración: Los ejemplares en alcohol son completamente blancos.

Aparato ocular: Ausente.

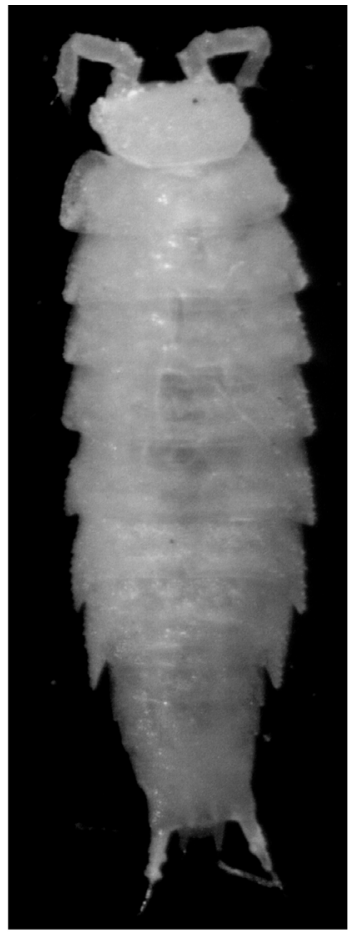

1

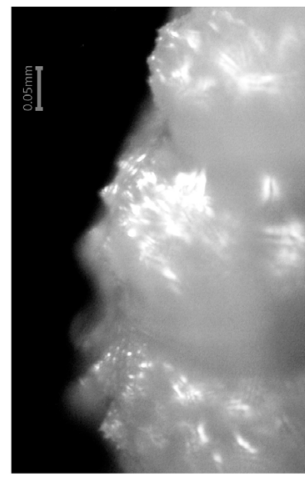

2

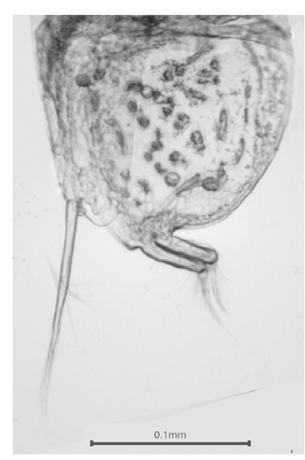

3
Caracteres tegumentarios: Tegumento recubierto de grandes escamas, y con fuertes granulaciones (Figs. 1-2), dispuestas en varias filas irregulares en el céfalon, en tres filas en el primer terguito del pereion y en dos filas en el resto. Estas granulaciones están coronadas por una seda, y se atenúan desde el céfalon al último pereonito, además las granulaciones de la fila posterior son más grandes que las del resto.

Apéndices: Anténula con tres estetascos largos en el tercer artejo. Antenas muy cortas, $\operatorname{los}$ artejos $4^{\circ}$ y $5^{\circ}$ son algo más cortos que el flagelo. Éste está formado por tres artejos, con un grupo de estetascos en el $2^{\circ}$.

Caracteres sexuales del macho:

- Pereiópodo $1^{\circ}$ con numerosas escamas hialinas en la cara interna del meropodito y del carpopodito.

- Pereiópodo $7^{\circ} \sin$ diferenciación sexual.

- Pleópodo I: El exopodito es cuadrangular, con el borde externo redondeado y el interno recto. Los dos tallos ciliados son casi iguales (Figs. 3, 7). El endopodito es triangular, con un lóbulo distal redondeado (Figs. 3, 8).

- $\quad$ Pleópodo II: El exopodito es triangular, con una punta interna muy desarrollada, provista de un fuerte tallo (Figs. 4, 9). El endopodito es recto (Figs. 5, 10), el artejo distal es el doble de la longitud del artejo basal, está rodeado de una membrana hialina y termina en una punta delgada (Fig. 6).
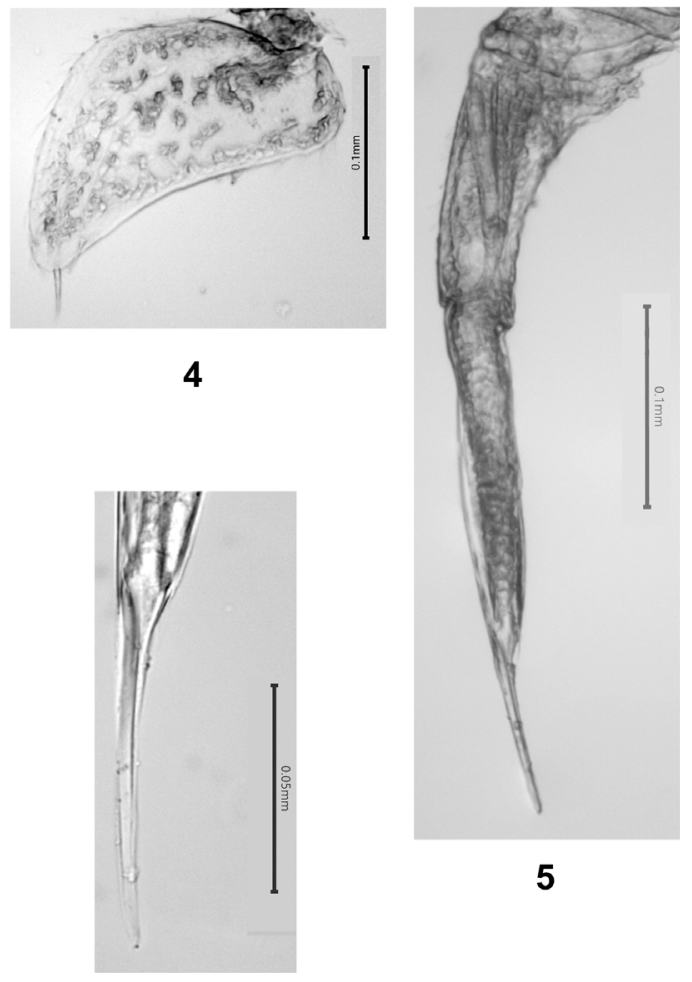

5

6

Figs. 1-6.- Trichoniscoides cantabricus n. sp.: 1: Habitus. 2: Granulaciones del $1^{\circ}, 2^{\circ}$ y $3^{\text {er }}$ terguito del pereion. 3: Exopodito y endopodito I $\delta$. 4: Exopodito II $\delta$. 5-6: Endopodito II $\partial$.

Figs. 1-6.- Trichoniscoides cantabricus n. sp.: 1: Habitus. 2: Granulations of the first, second and third tergites of the pereon. 3: Exopod and endopod I $\partial^{\lambda}$. 4: Exopod II $\partial^{2}$. 5-6: Endopod II $\partial^{2}$. 


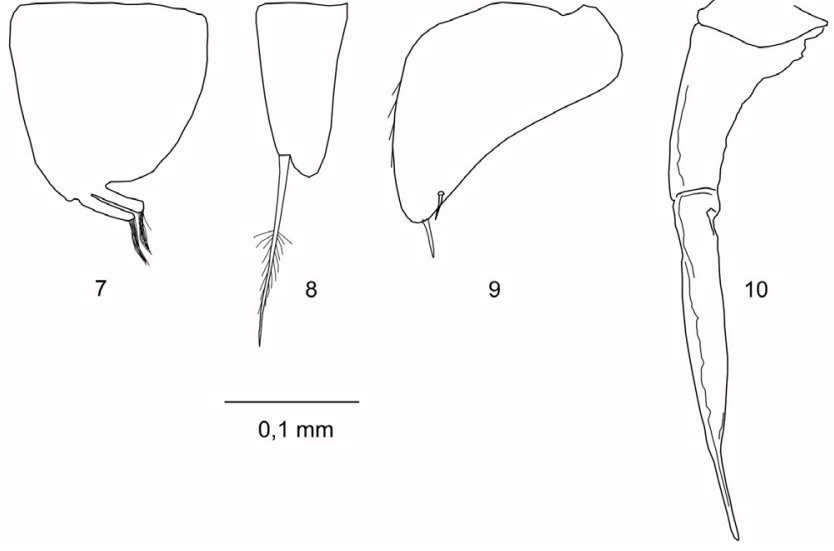

Figs. 7-10.- Trichoniscoides cantabricus n. sp.:

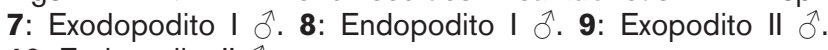
10: Endopodito II $\sigma^{\lambda}$.

Figs. 7-10.- Trichoniscoides cantabricus n. sp.: 7: Exopod I ${ }^{7}$. 8: Endopod I . 9: Exopod II $\partial$. 10: Endopod II $\partial$.

ECOLOGÍA Y DisTRIBUCIÓN GEOGRÁFICA. Se ha encontrado en catorce cavidades, en un rango altitudinal de 60-585 msm en Cantabria, y hasta 827 msm en Burgos, que se distribuyen geográficamente en cinco áreas kársticas. La central y más extensa incluye las montañas que conforman las cuencas medias de los ríos Asón y Miera; al oeste se encuentra en Puente Viesgo (cuevas Sumidero, Búho y, separada por el río Pas, Pis o Piz) y más al oeste en Udías, separada del área anterior por los ríos Saja y Besaya. Las otras dos áreas, separadas 20-25 km del área principal, están al Este (Castro Urdiales: La Lastrilla) y al sur (Merindad de Sotoscueva: Racino); esta última, ya en Burgos, está separada de la principal por la Sierra del Valnera, aunque su naturaleza calcárea y la falta de datos hacen plausible una conexión geográfica más estrecha (Fig. 32).

Como otras especies del género, los individuos se pueden localizar sobre las paredes muy húmedas aunque es más frecuente encontrarlos sobre trozos de madera en descomposición. En tres cavidades, Cova1larco, Molino y Pis, coexiste con T. cavernicola. Es de descatar el hecho de que hasta ahora no se había mencionado la coexistencia en la misma cavidad de dos especies de Trichoniscoides.

\section{Troglonethes fonsocalvoi $\mathrm{n}$. sp.}

urn:Isid:zoobank.org:act:0DA3B9E0-2D86-4140-9E10-77629C259AF2

Material típico. Holotipo, đ̂, Bizkaia, Galdames, Hoyo Ganerán (30TVN9322990577, 593 msm), 28-VI-2013, Alfonso Calvo leg., MNCN 20.04/12332 [ex ZUPV 1271].

Paratipos: Álava: Gopegi, cueva de Peña Gingia (30TWN2060261678, 711 msm), 21-IV-2013, 1 ㅇ, C. Prieto, T.L. Ipiña y G. Leanizbarrutia leg., ZUPV 1039. Izarra, Sierra de Gibijo, torca de Lejazar (30TWN0303153579, $836 \mathrm{msm}$ ), 28-XII-2013, 1 ઈิ y 1 क , C. Prieto, M. Vila, A. Calvo, I. Robles leg., ZUPV 1743. Bizkaia: Alonsotegi, Zaramillo, mina Amalia (30TVN9840686965, 80 msm), 26-VI-2017, 1 ô y 1 + , A. Calvo leg., ZUPV 4933. Alonsotegi, Zamundi, cueva-mina de Castañofil (30TVN9898488453, 299 msm), 12-XI-2015, 1 ㅇ, A. Calvo leg., ZUPV 3225. Arcentales, mina del Pedreo (30TVN8263190598, 440 msm), 22-II-2014, 1 ð̊ y 1 \& , C. Prieto, A. Calvo y P. Jiménezleg., ZUPV 1947. Galdames, cueva de Arenaza (30TVN9189789644, 195 msm), 24-IX-2014, 1 సै y 1 ㅇ, A. Calvo leg., ZUPV 2615; 21-09-2019, 1 §., A. Calvo leg., ZUPV 5713. Galdames, sima Eskatxabel 2 (30TVN9279491367, $570 \mathrm{msm}$ ), 31-III-2013, 1 ठ y 1 , , C. Prieto y A. Calvo leg., ZUPV 1001. Galdames, soplado de Eskatxabel (30TVN9270491265, 560 msm), 17-V-2012, 1 के y 3 우, C. Prieto, Jon Fernández y A. Calvo leg., ZUPV 729. Galdames, mina Europa (= La Fragua) (30TVN9295089225, $350 \mathrm{msm}$ ), 6-VI-2010, 1 đ̂ y 3 + 9 , C. Prieto, J. MorenoyA.Calvo leg., ZUPV 506, 507. Galdames, hoyo Ganerán (30TVN9322990577, 593 msm), 15-XI-2014, 1 ㅎ y 3 우오. A. Calvo leg., ZUPV 2646. Galdames, cueva del Hoyo (30TVN9218489712, $315 \mathrm{msm}$ ), 2-XI-2014, 1 \&, A. Calvo leg., ZUPV 2641. Galdames, soplado de La Hiedra (30TVN9296590886, $511 \mathrm{msm}$ ), 15-VII-2013, 1 오, A. Calvo leg., ZUPV 1320; 24-VII-2013, 1 ô y 6 우, C. Prieto y A. Calvo leg., MNCN 20.04/12333 [ex ZUPV 1353]. Galdames: Cueva de La Magdalena (30TVN9204991222, 475 msm), 11-VII1982, 1 ô, J. Riezu leg., ZUPV 2402; 30-VII-2013, 1 , C. Prieto y A. Calvo leg., ZUPV 1393. Galdames, soplado de mina Europa (30TVN9305089225, 350 msm), 6-XII-2013, 1 ๙ิ y 1 \& , A. Calvo leg., ZUPV 1710; 1-I-2015, 1 \% , A. Calvo leg., ZUPV 2690; 30X-2016, 1 , A. Calvo leg., ZUPV 4220. Galdames, soplado de mina Prado de Artekona (30TVN9271090156, $451 \mathrm{msm}$ ), 25-X2014, 2 우, A. Calvo leg., ZUPV 2636. Galdames, soplado de mina Princesa (30TVN9286791182, $567 \mathrm{msm}), 17-\mathrm{V}-2012,1$ ô, C. Prieto, A. Calvo y J. Fernández leg., ZUPV 734. Galdames, sumidero Rosario-4,6 (30TVN9358089859, 659 msm), 13-IX2014, 1 §ै, A. Calvo leg., ZUPV 2613; 11-X-2014, 1 ㅇ, A. Calvo leg., ZUPV 2632; 2-VIII-2015, 2 우, A. Calvo leg., ZUPV 2952. Güeñes, cueva del Grazal (30TVN9535984852, 148 msm), 3-VII2013, 1 ㅇ, C. Prieto y A. Calvo leg., ZUPV 1286; 02-X-2016, 3 우우, C. Prieto y J. Fernández leg., ZUPV 4865. Orozko, Anuntzibai, sistema de Erreketako (30TWN0611676220, $221 \mathrm{msm}$ ), 16-VII2014, 4 ठิ ठิ y 3 우, C. Prieto, Iker y Eider leg., ZUPV 2498. Orozko, Itxina, cueva de Supelegor (30TWN1504569091, $1000 \mathrm{msm}$ ), 9-XI-1980, 1 ठ̊, C. Prieto leg., ZUPV 14 [publicada por Vivar et al., 1984 como T. cavernicola]. Orozko, cueva Urratxa (30TWN1641665947, 1006 msm), 11-VII-2015, 1 กे y 1 ㅇ, C. Prieto y O. Gorosabel leg., ZUPV 2889. Ortuella, cueva de la Arboleda 1 (30TVN9516491922, 464 msm), 1-VI-1973, 1 , G.E. Esparta leg., ZUPV 2156. Trucíos, Basinagre, torca de la Campa Loredo (30TVN7921991956, $207 \mathrm{msm}), 1$-VII-2011, 1 §ิ, J. Moreno leg., ZUPV 697. Valle de Trápaga, mina Oculta

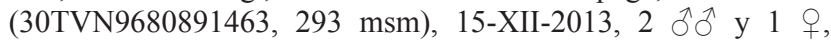
C. Prieto y A. Calvo leg., ZUPV 5700. Valle de Trápaga, La Arboleda, mina Precavida (30TVN9609691890, 370 msm), 27IX-2015, 2 đ̋ sima MS-3 (30TWN0015454256, 820 msm), 12-V-2013, 2 우우, C. Prieto e I. Robles leg., ZUPV 1085; 28-XII-2013, 1 đ̊ y 1 으, C. Prieto, M. Vila, A. Calvo e I. Robles leg., ZUPV 1749. Villalba de Losa, cueva de Albia (30TVN9396557332, 806 msm), 4-IX-

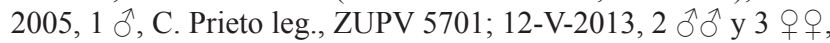
C. Prieto leg., ZUPV 1097; 19-II-2017, 3 ठे $\widehat{\jmath}$ y 1 क , C. Prieto y J. Fernández leg., ZUPV 4486. Villalba de Losa, cueva del Puente (30TVN9461760300, $1011 \mathrm{msm}$ ), 9-VI-2013, 1 ô, C. Prieto, J. Fernández e I. Robles leg., ZUPV 1197. Cantabria. Guriezo, Llaguno, cueva-sumidero de El Jorrazo (30TVN7816494229, $220 \mathrm{msm}), 17-\mathrm{X}-2015,1$ §̊, C. Prieto y A. Calvo leg., ZUPV 3051.

Se han estudiado 84 ejemplares, 34 de los cuales son machos (40\%) y 50 son hembras. 
Etimología. El nombre de la especie es un reconocimiento a la labor bioexploradora de Alfonso Calvo, espeleólogo de la Sociedad Espeleológica Burnia.

Diagnosis. Tegumentos con granulaciones. Antenas largas con el flagelo antenal formado por 5 artejos. Tres primeros pares de pereiópodos del macho con escamas hialinas en el meropodito y el carpopodito. Pereiópodo 7 del macho con diferenciación sexual, consistente en un fuerte saliente provisto de numerosas escama en la cara dorsal del carpopodito. El endopodito del pleópod II del macho es biarticulado con un gancho poco marcado en el ápice formado por dos estructuras cónicas que soportan una lámina hialina.

\section{DESCRIPCIÓN}

Talla máxima observada: $4 \times 1,6 \mathrm{~mm}$ sin los uropodos.

Coloración: Los ejemplares en alcohol son completamente blancos.

Aparato ocular ausente.

Mandíbula izquierda con tres tallos ciliados, mandíbula derecha con un tallo ciliado.
Caracteres tegumentarios: En el céfalon presenta varias filas irregulares de granulaciones. En el pereion hay dos filas, las granulaciones de la fila anterior se atenúan hacia la región posterior, mientras que las de la segunda fila son muy grandes en todo el pereion. El pleon y el telson no tienen granulaciones (Fig. 11).

Apéndices: Anténula con un grupo de cuatro gruesos estetascos en el tercer artejo (Figs. 12, 22). Antenas largas, con el $3^{\circ}, 4^{\circ}$ y $5^{\circ}$ artejos con tubérculos escamosos. El flagelo consta de 5 artejos, con grupos de dos cortos estetascos en el segundo, tercero y cuarto artejos (Figs. 13, 23).

Caracteres sexuales del macho:

- Pereiópodos $1^{\circ}, 2^{\circ}$ y $3^{\circ}$ con numerosas escamas hialinas en la cara interna del meropodito y del carpopodito (Figs. 14-15, 24), ausentes en la hembra.

Pereiópodo 7: El carpopodito presenta un fuerte saliente en la cara dorsal, provisto de numerosas escamas (Figs. 16-17, 25).

Apófisis genital muy larga, gruesa y terminada en punta roma (Fig. 26).

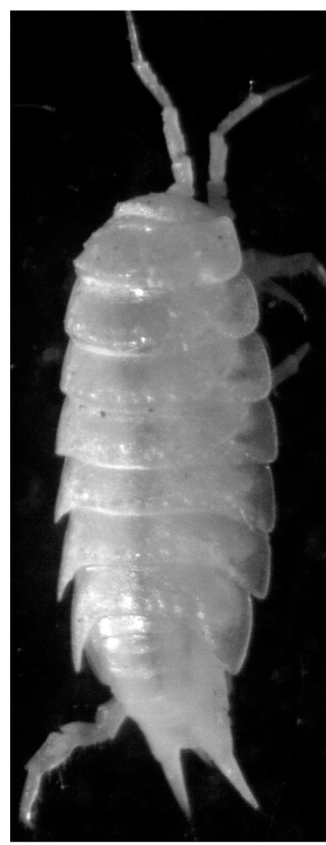

11

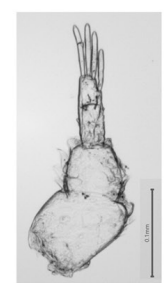

12

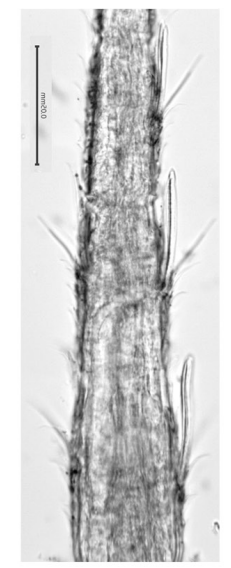

13

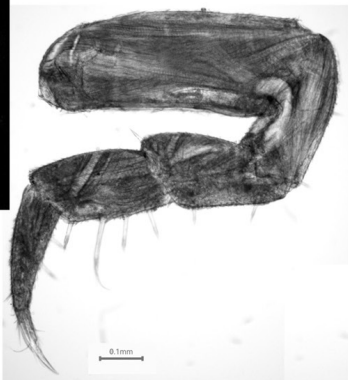

14

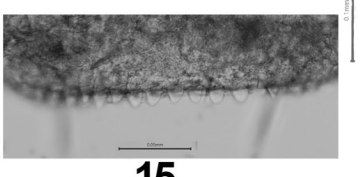

15

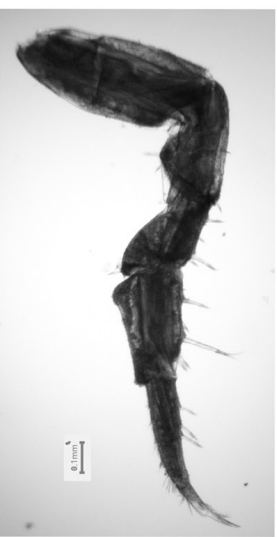

16

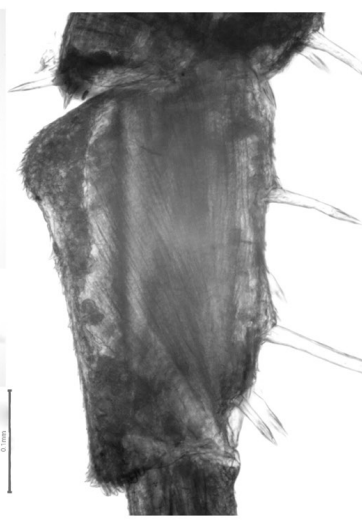

17

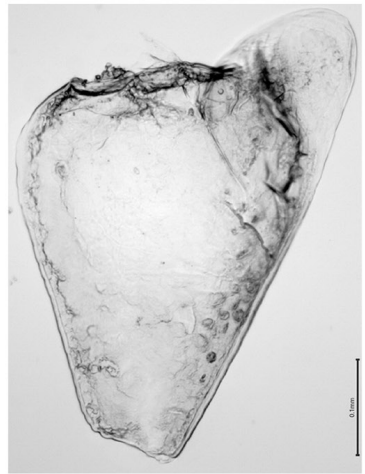

18

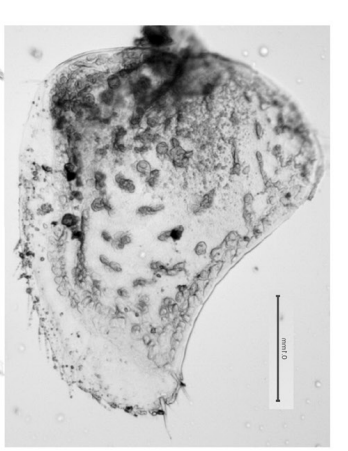

19

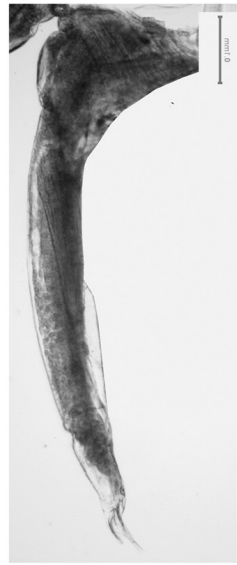

20

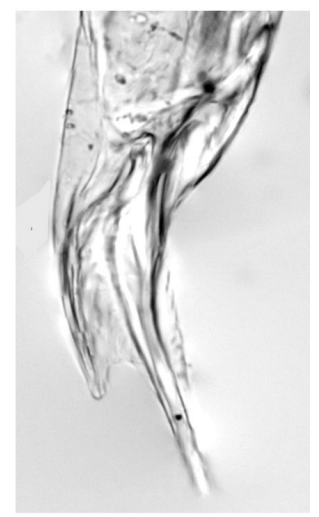

21

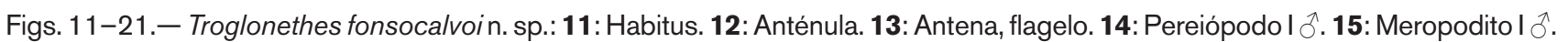

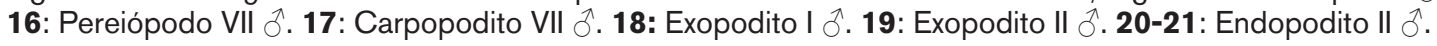

Figs. 11-21.- Troglonethes fonsocalvoi n. sp.: 11: Habitus. 12: Antennula. 13: Artenna, flagellum. 14: Pereopod I $\delta$. 15: Meros I $\delta$.

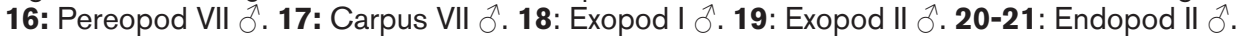


- Pleópodo I: El exopodito es triangular, casi el doble de largo que ancho, con el extremo truncado, aunque en algunos ejemplares puede ser redondeado (Figs. 18, 27). El endopodito es triangular con el borde externo sinuoso (Figs. 28); el artejo basal es mucho más corto que el exopodito.

- $\quad$ Pleópodo II: El exopodito es triangular, casi tan largo como ancho y con el extremo redondeado provisto de dos fuertes tallos acompañados de numerosasas sedas; el borde interno está fuertemente curvado y provisto de grupos de sedas en peine; el borde externo es cóncavo y presenta un ángulo basal fuertemente redondeado (Figs. 19, 29). El endopodito es biarticulado, con el artejo basal más de tres veces la longitud del artejo distal; con un gancho poco marcado en el ápice formado por dos estructuras cónicas que soportan una lámina hialina (Figs. 20-21, 30-31).

Ecología. Se ha encontrado en 29 cavidades, en un rango altitudinal de $80-1010 \mathrm{msm}$, que se distribuyen geográficamente en cuatro áreas kársticas. La localidad típica y la mayoría de localidades (entre 80 y 659 msm, media 402) se concentran en los Montes de Triano, macizo situado al Oeste de Bilbao y

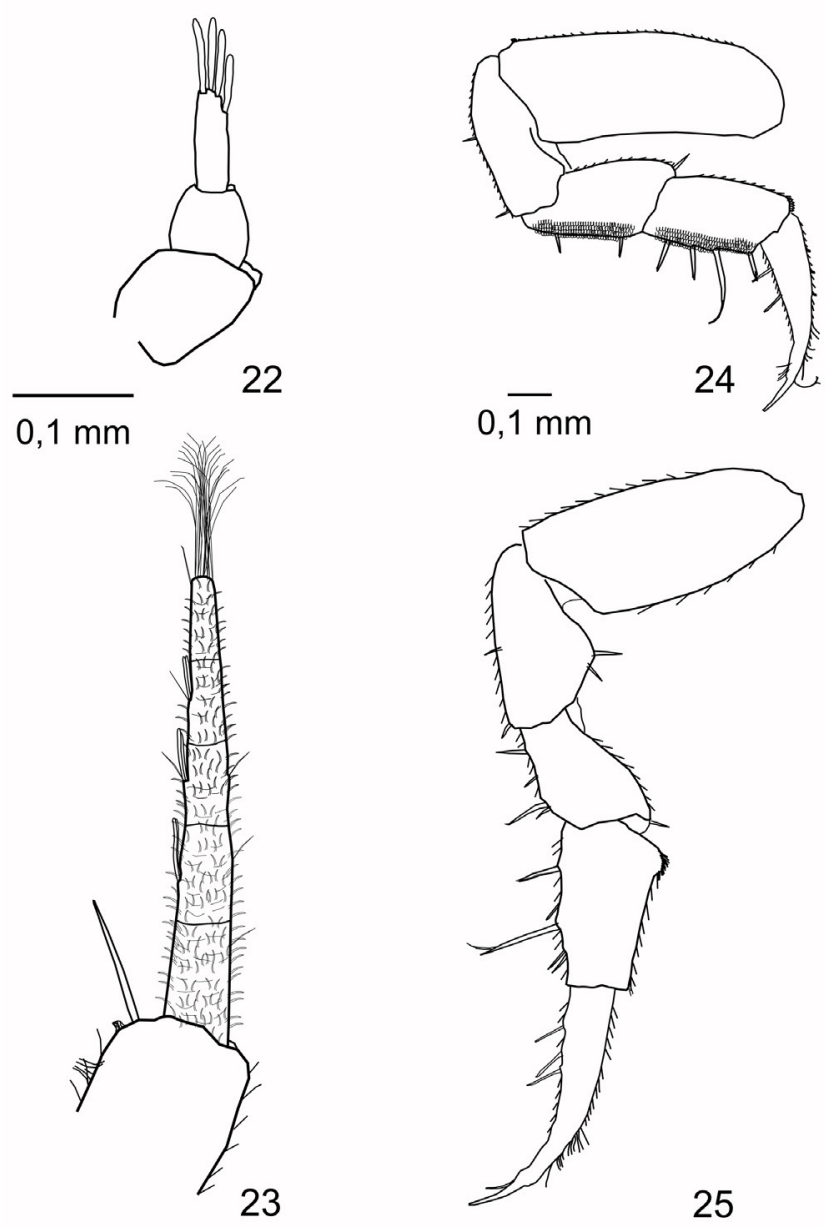

Figs. 22-25.- Troglonethes fonsocalvoi n. sp.: 22: Anténula. 23: Antena, flagelo. 24: Pereiópodo I $\delta$. 25: Pereiópodo VII $\widehat{\partial}$.

Figs. 22-25.- Troglonethes fonsocalvoi n. sp.: 22: Antennula. 23: Artenna, flagellum. 24: Pereopod I $\hat{\delta}$. 25: Pereopod VII $\hat{\partial}$.

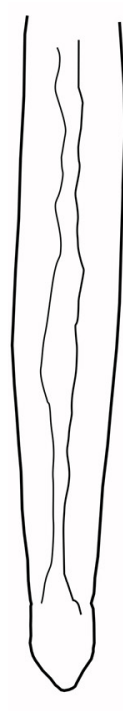

26

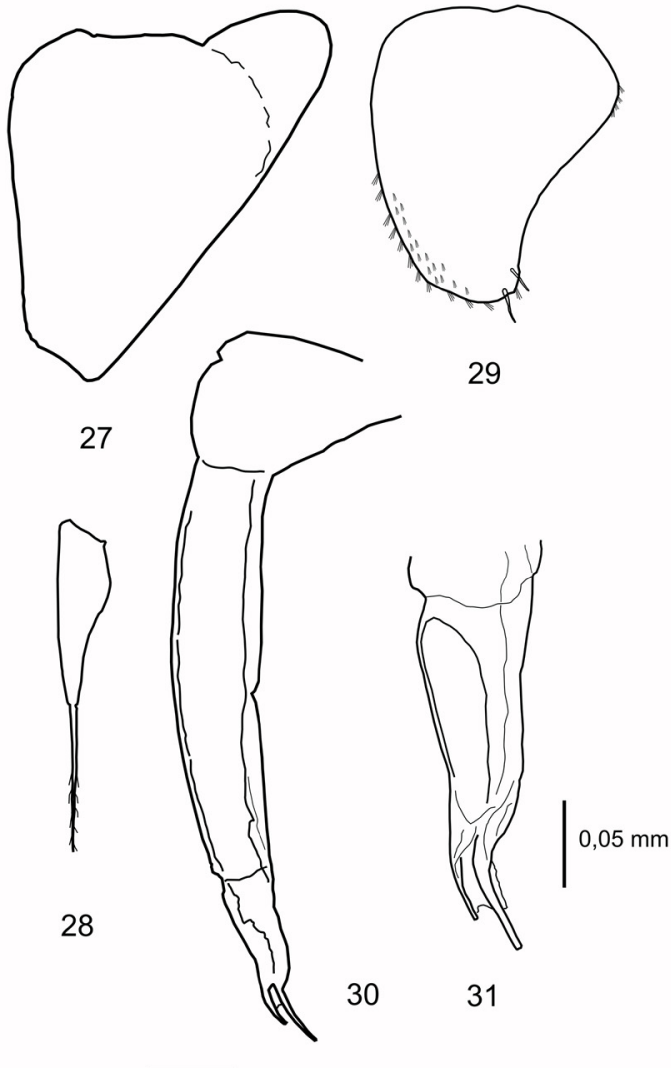

$0,1 \mathrm{~mm}$
Figs. 26-31.- Troglonethes fonsocalvoi n. sp.: 26: Apófisis genital. 27: Exopodito I $\sigma^{\lambda}$. 28: Endopodito I $\partial^{\lambda}$. 29: Exopodito II ${ }^{\lambda}$. 30-31: Endopodito II $\delta$.

Figs. 26-31.- Troglonethes fonsocalvoi n. sp.: 26: Genital papilla. 27: Exopod I $\delta$. 28: Endopod I $\delta$. 29: Exopod II $\delta$. 3031: Endopod II 0 .

donde está presente en sus tres vertientes. Al Oeste, y separada por el río Mercadillo, se encuentra en el complejo Alén-Jorrios, atravesado por el río Agüera (rango, 207-440 msm). Al Sur vive en dos macizos netamente diferenciados, Sierra Salvada entre Álava y Burgos (rango, 806-1011 msm), y Macizo de Gorbea entre Bizkaia y Álava (rango, 711-1006 msm), al cual se podría adscribir la cueva de Erreketako, situada a $220 \mathrm{msm}$ y a mitad de distancia con Montes de Triano (Fig. 32).

El conjunto de las poblaciones meridionales ( $>700 \mathrm{msm})$ presenta un sex-ratio de 0,5 (10 machos y 10 hembras) mientras que en el de las septentrionales (<700 msm) es de 0,35 (20 machos y 37 hembras).

Los individuos se pueden localizar tanto sobre paredes muy húmedas, especialmente si están cubiertas por arcilla y más frecuentemente sobre trozos de madera en descomposición. En las cavidades de Sierra Salvada coexiste con Cantabroniscus primitivus (datos inéditos), aunque esta especie tiene un nicho ecológico diferente por estar restringida a ambientes acuáticos. 


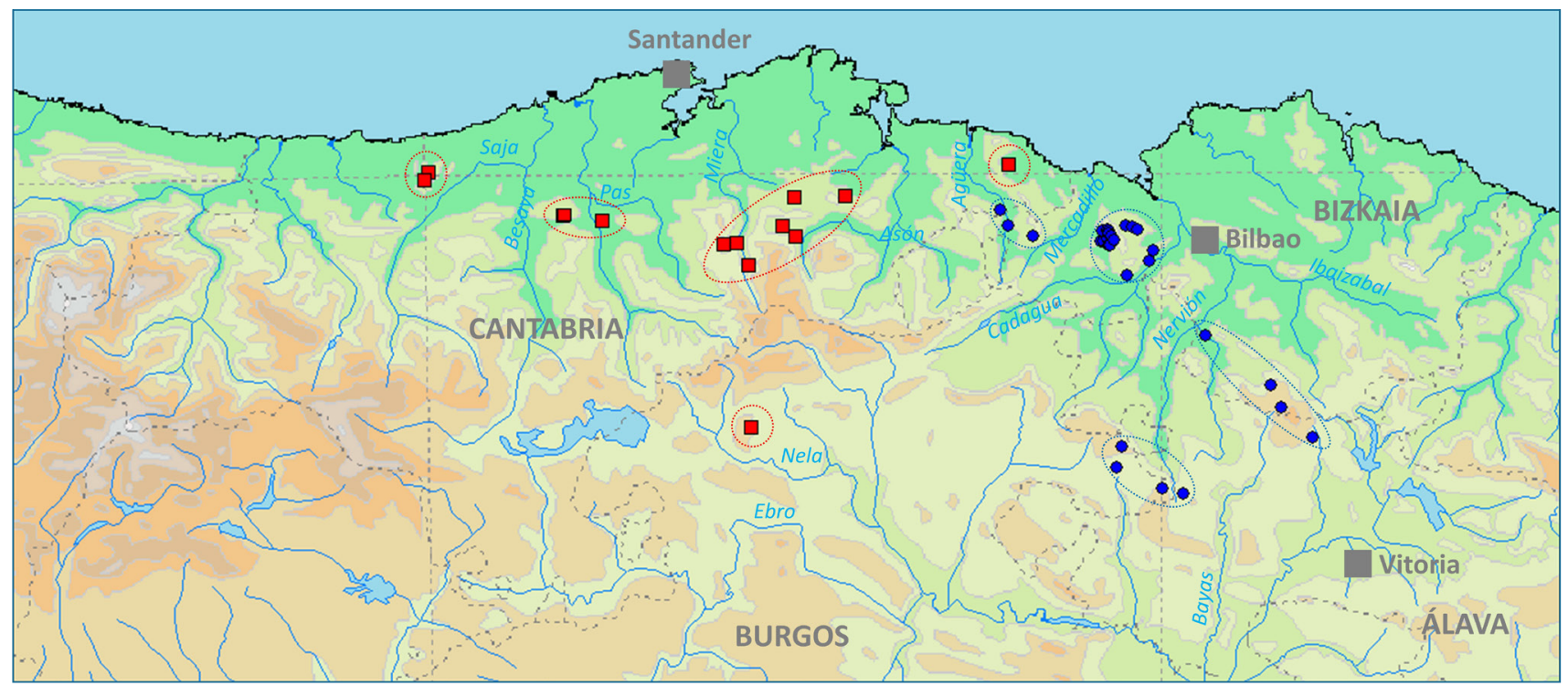

Fig. 32.- Distribución geográfica conocida de Trichoniscoides cantabricus n.sp. (cuadrados rojos) y Troglonethes fonsocalvoi n. sp. (círculos azules).

Fig. 32.- Known geographical distribution of Trichoniscoides cantabricus n.sp. (red squares) and Troglonethes fonsocalvoi n. sp. (blue dots).

\section{Discusión}

La estructura del endopodito del segundo pleópodo del macho de Trichoniscoides cantabricus n. sp., sitúa a esta nueva especie en el grupo "atlántico" de Vandel (1946, 1952, 1960), y además su tegumento presenta grandes granulaciones. En el mismo grupo, también presentan el tegumento granuloso T. albidus (BuddeLund, 1880); T. bellesi Reboleira \& Taiti, 2015; T. breuilli Vandel, 1952; T. broteroi Vandel, 1946; T. marinae Cifuentes, 2019; T. meridionalis Vandel, 1946, T. modestus Racovitza, 1908; T. ouremensis Vandel, 1946; T. pitarquensis Cruz, 1993; T. saeroeensis Lohmander, 1924 y T. serrai Cruz, 1993.

Dado que ya se han descrito los caracteres importantes que permiten su separación específica, a continuación se indicarán algunos caracteres que permiten diferenciar a $T$. cantabricus del resto de las especies mencionadas del grupo "atlántico" que presentan el tegumento granuloso. T. breuilli tiene un tallo ciliado en el exopodito I del macho muy desarrollado y característico, frente a los dos tallos más pequeños de T. cantabricus. T. bellesi, T. meridionalis, T. ouremensis y $T$. pitarquensis carecen de un lóbulo marcado en el endopodito I del macho, que sí presenta $T$. cantabricus. T. serrai y T. broteroi presentan un entrante en la base de los tallos ciliados del exopodito I del macho, y $T$. broteroi también presenta una estructura en forma de gancho en el meropodito del séptimo pereiópodo del macho, y ninguna de esas dos características se presenta en la nueva especie. T. marinae y T. saeroeensis presentan los tallos del exopodito I del macho cortos, mientras que están bien desarrollados en $T$. cantabricus, además los endopoditos II del ma- cho son muy diferentes en las tres especies. T. albidus presenta unos largos tallos ciliados en el exopodito I del macho y un gran lóbulo en el artejo basal del endopodito I del macho, mientras que en T. cantabricus, los tallos ciliados son más pequeños así como el lóbulo del endopodito I. También T. $s p$. $B$ de Gregory et al. (2012) tiene un gran lóbulo triangular en el artejo distal del endopodito I del macho, y además los dos tallos ciliados del exopodito I del macho son muy desiguales, siendo el interno más pequeño que el externo, caracteres ambos que no se encuentran en T. cantabricus, ya que el lóbulo es más pequeño y redondeado y los tallos son poco desiguales. Y finalmente, T. modestus es una especie de Francia, que presenta el endopodito II del macho sin eje quitinoso y con una lámina hialina de desarrollo variable según zonas (Vandel, 1952, 1960), y con el artejo distal hasta cuatro veces más largo que el proximal, mientras que este artejo es mucho más corto en $T$. cantabricus, además de presentar una lámina hialina.

En cuanto a Troglonethes fonsocalvoi n. sp., según Vandel (1960), la segunda legión de la subfamilia Trichoniscinae Racovitza, 1908 agruparía a formas primitivas por presentar escasa diferenciación sexual a nivel de los pleópodos I y II del macho. Esta legión está representada en la península ibérica por cuatro géneros: Alpioniscus Racovitza, 1908; Libanonethes Vandel, 1955; Spelaeonethes Verhoeff, 1932 y Troglonethes Cruz, 1989. Estos cuatro géneros pueden separarse mediante la clave que facilita Cruz (1989) al describir el género y la especie $T$. aurouxi.

Actualmente el género Troglonethes esta formado por cuatro especies endémicas de la península ibérica, T. aurouxi descrita de Cova de les Aranyes en Valencia, 
de la cual hemos podido estudiar los ejemplares tipo; T. olissipoensis Reboleira \& Taiti, 2015 descrita de la Gruta da Alvide en Cascais en el distrito de Lisboa en Portugal (Reboleira et al., 2015); T. arrabidaensis Reboleira \& Taiti, 2015 descrita de la Gruta do Frade, Arrábida Massif en el distrito de Sétubal también en Portugal (Reboleira et al., 2015) y la que se describe en este trabajo, T. fonsocalvoi n. sp. de numerosas cavidades de Araba, Bizkaia, Burgos y Cantabria en España.

$\mathrm{Si}$ bien las cuatro especies presentan una estructura parecida en la forma del exopodito I del macho, acorde con la escasa diferenciación sexual que caracteriza a la segunda legión de Vandel (1960), en las dos especies de Portugal, este exopodito es tan largo como ancho, mientras que en las dos especies de España, el exopodito es más largo que ancho. Sin embargo las diferencias más notables se observan en la diferenciación sexual de los pereiópodos del macho, con brochas de sedas hialinas en el meropodito y carpopodito de los tres primeros pares en $T$. fonsocalvoi que no existen en el resto de las especies, y un fuerte saliente en la base del lado tergal del carpopodito 7 del macho de T. arrabidaensis y T. fonsocalvoi. También se observan diferencias importantes en la estructura del endopodito II del macho, así en T. olissipoensis el artejo basal es tres veces más largo que el distal y éste presenta un gancho en la base y otro muy largo en el ápice. En T. aurouxi y T. arrabidaensis el artejo basal es cerca de dos veces y media la longitud del distal y presenta un único y largo gancho en el ápice, mientras que en $T$. fonsocalvoi el artejo basal es muy largo, más de tres veces la longitud del artejo distal, el gancho del ápice está poco desarrollado y está formado por dos estructuras cónicas que soportan una lámina hialina (Figs. 20-21, 30-31).

Otros caracteres que se pueden utilizar para la ìdentificación específica de estas cuatro especies son, el número de estetascos en la anténula, cuatro en $T$. fonsocalvoi y tres en el resto. El número de artejos en el flagelo antenal, tres en T. arrabidaensis, cuatro en $T$. aurouxi y cinco en T. arrabidaensis y $T$. fonsocal$v o i$, aunque en esta especie hay tres grupos de estetascos frente a los dos de T. arrabidaensis.

Con objeto de facilitar la identificación de las especies de Troglonethes, se facilita una clave que solamente es aplicable a los machos.

1 Pereiópodo 7 semejante en ambos sexos ....................2

- Pereiópodo 7 del macho con diferenciación sexual ....3

2 Artejo distal del endopodito del pleópodo II con un único gancho en posición distal

T. aurouxi Cruz, 1989

- Artejo distal del endopodito del pleópodo II con un gancho basal y otro distal

T. olissipoensis Reboleira \& Taiti, 2015

3 Pereiópodo I sin diferenciación; flagelo antenal con 3 artejos

T. arrabidaensis Reboleira \& Taiti, 2015

- Pereiópodo I con escamas hialinas; flagelo antenal con 5 artejos

\section{Agradecimientos}

Queremos expresar nuestro agradecimiento a los diferentes recolectores por su ayuda en la captura de los ejemplares utilizados para describir las nuevas especies, y especialmente a Alfonso Calvo y Jon Fernández. A la Dra. Begoña Sánchez, conservadora del Museo Nacional de Ciencias Naturales de Madrid, al Dr. Antoni Serra Sorribes del Centre de Recursos de Biodiversitat Animal de la Universitat de Barcelona y a Glòria Masó, conservadora de la colección de artrópodos del Museo de Barcelona por su ayuda para la consulta de las respectivas colección de isópodos, que nos ha brindado la posibilidad de comparar los ejemplares de las nuevas especies con los de otras especies depositadas en las colecciones de los citados museos. Al Dr. Ernesto Recuero y a un revisor anónimo por sus valiosos comentarios que han ayudado a mejorar este trabajo.

\section{Referencias}

Bellés Ros, X., 1987. Fauna cavernícola i intersticial de la Península Ibèrica $i$ les Illes Balears. Monografies Científiques, 4. CSIC \& Ed. Moll, Palma de Mallorca. 207 pp.

Cifuentes, J., 2019. Contribution à la connaissance des Trichoniscoides Sars, 1899 ibériques, avec la description de deux nouvelles espèces: Trichoniscoides marinae $\mathrm{n}$. $\mathrm{sp}$. et Tr. enoli n. sp (Crustacea, Isopoda, Trichoniscidae). Boletín de la Asociación española de Entomología, 43 (1-2): 27-54.

Cruz, A., 1989. Troglonethes aurouxi gen. n., sp. n. (Oniscidea Trichoniscidae), un isópodo terrestre cavernícola del Levante español. Miscel·lània Zoològica, 13: 51-54.

Gregory, S., Lee, P., Read, H. J. \& Richards, P., 2012. Woodlice (Isopoda: Oniscidea) collected from northwest Spain and northern Portugal in 2004 by the british myriapod and isopod group. Bulletin of the British Myriapod \& Isopod Group, 26: 6-23.

Reboleira, A. S., Gonçalves, F., Oromí, P. \& Taiti, S., 2015. The cavernicolous Oniscidea (Crustacea: Isopoda) of Portugal. European Journal of Taxonomy, 161: 1-61. https://doi.org/10.5852/ejt.2015.161

Schmalfuss, H., 2003. World catalog of terrestrial isopods (Isopoda: Oniscidea). Stuttgarter Beiträge zur Naturkunde, Serie A, 654: 1-341. Disponible en http:// www.oniscidea-catalog.naturkundemuseum-bw.de/ (consultado 14 Sep. 2020).

Schmölzer, K., 1971. Die Landisopoden der Iberischen Halbinsel. Monografías de Ciencia moderna $n^{\circ} 80$. Instituto José de Acosta, CSIC. XI + 161 pp., 10 maps.

Taiti, S., 2004. Crustacea: Isopoda: Oniscidea (Woodlice). In: J. Gunn (Ed.). Encyclopedia of Caves and Karst Science. Taylor \& Francis, London: 547-551.

Vandel, A., 1946. Crustacés isopodes terrestres (Oniscoïdea) épigés et cavernicoles du Portugal. Anaïs da Faculdade de Ciências do Porto, 30: 135-427.

Vandel, A., 1952. Biospeologica LXXIII. Isopodes terrestres (troisième série). Archives de Zoologie expérimentale et générale, 88: 231-362.

Vandel, A., 1960. Isopodes terrestres (première partie). Faune de France, 64: 1-416. Paul Lechevalier. Paris. 